\title{
Diseño de una metodología gerencial para proyectos de sistemas distribuidos de antenas*
}

[Artículos de investigación]

\author{
Yira Liliana Buelvas Osorio ${ }^{* *}$
}

Recibido: 17 de febrero de 2020

Revisado: 12 de agosto de 2020

Aceptado: 14 de agosto de 2020

Cómo citar este artículo:

Buelvas Osorio, Y. L. (2020). Diseño de una metodología gerencial para proyectos de sistemas distribuidos de antenas. Signos, Investigación en sistemas de gestión, 13(1). https://doi.org/10.15332/24631140.6341

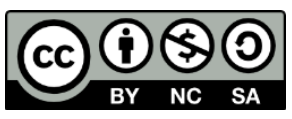

\section{Resumen}

Este artículo brinda orientaciones a los gerentes de proyectos para diseñar una metodología gerencial que facilite la ejecución de sistemas distribuidos de antenas de tecnología $3 \mathrm{G}$ en espacios indoor. La metodología cuantitativa exploratoria permitió un análisis concreto como base para la propuesta del diseño. Así, se determinó el estándar más adecuado en la dirección de proyectos para este tipo de soluciones y,

\footnotetext{
*Artículo de resultado de investigación.

** Universidad Militar Nueva Granada. Magíster en Gerencia de Proyectos. Ingeniera de Telecomunicaciones. Línea de investigación: Metodologías Gerenciales. Universidad Militar Nueva Granada, Bogotá, Colombia. Correo electrónico: U21100140@unimilitar.edu.co, yirabuelvasosorio@gmail.com. ORCID: https://orcid.org/0000-0002-8346-8236. CvLAC: https://scienti.minciencias.gov.co/cvlac/visualizador/generarCurriculoCv.do?cod_rh $=0$ $\underline{001815588}$
} 
teniendo como referentes los resultados del instrumento aplicado, se diseñó una metodología a la medida en la que solo se intervienen las áreas críticas. El uso de esta metodología disminuye costos y tiempos en todas las fases, brinda satisfacción final al cliente y permite monitorear el progreso de cada tarea a lo largo de la implementación; en general, es una herramienta útil que facilita el seguimiento durante la ejecución y la finalización del proyecto. Se concluye que las buenas prácticas y la aplicación de una metodología gerencial permiten, en gran medida, el logro de los objetivos.

Palabras clave: sistemas distribuidos de antenas, telecomunicaciones, $3 \mathrm{G}, \mathrm{RF}$, metodología, gestión de proyectos.

\section{Design of a management methodology for distributed antenna system projects}

\section{Abstract}

This article provides guidance to project managers to design a management methodology to facilitate the implementation of distributed systems of $3 \mathrm{G}$ technology antennas in indoor spaces. The quantitative exploratory methodology allowed a concrete analysis as a basis for the design proposal. Thus, the most appropriate standard in project management for this type of solution was determined; taking as a reference the applied instrument results, a custom-made methodology was designed, and only critical areas were involved. Using this methodology reduces costs and time in all phases, provides final satisfaction to the client and allows monitoring the progress of each task throughout the implementation stage; in general, it is a useful tool that enable in-execution follow-up and project completion. It is then concluded that good practices and a management methodology application allow to a great extent the achievement of the objectives.

Keywords: distributed antenna systems, telecommunications, $3 \mathrm{G}, \mathrm{RF}$, methodology, project management. 


\section{Projeção de uma metodologia de gestão para projetos de sistemas de antenas distribuídos}

\section{Resumo}

Este artigo fornece orientação para os gerentes de projeto projetarem uma metodologia de gestão para facilitar a implementação de sistemas distribuídos de antenas de tecnologia $3 \mathrm{G}$ em espaços internos (indoor). A metodologia exploratória quantitativa permitiu uma análise específica como base para a proposta da projeção. Dessa forma, foi determinado o padrão mais adequado na gestão de projetos para esse tipo de solução; considerando como referência os resultados do instrumento aplicado, foi projetada uma metodologia personalizada e apenas áreas críticas foram envolvidas. O uso desta metodologia reduz custos e tempo em todas as fases, fornece satisfação final ao cliente e permite monitorar o progresso de cada tarefa ao longo da implementação; em geral, é uma ferramenta útil que facilita o acompanhamento durante a execução e conclusão do projeto. Conclui-se que as boas práticas e a aplicação de uma metodologia de gestão possibilitam, em grande parte, a realização dos objetivos.

Palavras-chave: sistemas de antenas distribuídos, telecomunicações, $3 \mathrm{G}, \mathrm{RF}$, metodologia, gestão de projetos.

\section{Introducción}

En los últimos años, se ha expandido el interés de distintas empresas por participar en el mercado de las telecomunicaciones, lo que se ha manifestado en el aumento de los operadores móviles y en las inversiones. Por su parte, las expectativas de los usuarios están orientadas al incremento de la calidad del servicio y la disminución de los precios. En este sentido, la Comisión de Regulación de Comunicaciones (CRC) ha desempeñado un papel vital en su objetivo de promover la protección de 
los derechos de los usuarios, la competencia en el mercado, la inversión en la infraestructura y la calidad de los servicios.

En Colombia, la telefonía celular ha evolucionado gradualmente en tecnologías 3G y 4G. Para soportar este crecimiento, se hace necesaria la expansión de infraestructura que permita el aprovisionamiento de dichas tecnologías, la ampliación de cobertura, mejores velocidades de datos, y, en general, una apropiada calidad al momento de la prestación del servicio. En esta ampliación, los sistemas distribuidos de antenas se presentan como una alternativa para proveer cobertura y capacidad adicional dentro de un área específica. Esta solución puede ser implementada en contextos que facilitan la concentración de gran cantidad de personas, tales como edificios, centros comerciales, bodegas y hospitales.

En el país, los sistemas distribuidos de antenas tienen un gran potencial de crecimiento debido a dos situaciones. En primer lugar, la participación de abonados, según datos estadísticos de la CRC, en el segundo trimestre del año 2019 fue de 62086 397, de los cuales solo el 50 \% tuvo acceso a internet móvil mediante la red $3 \mathrm{G}$ (Neira, 2019). En segundo lugar, en promedio se construyen 11 centros comerciales al año en Colombia, donde se requieren los servicios de telefonía celular teniendo en cuenta la concentración de un gran número de usuarios y la baja penetración de las señales de estaciones outdoor. Este tipo de proyectos de modernización está en auge en el país y el éxito de su implementación dependerá de la capacidad de los operadores móviles para aplicar buenas prácticas que permitan controlar el alcance, el cronograma y los costos, y mitigar los riesgos en el desarrollo de cada una de las fases de implementación. La gestión exitosa de los proyectos de implementación tecnológica ha sido uno de los objetivos más anhelados por todas las organizaciones, por lo que este tema ha llamado la atención de los investigadores en los últimos 
20 años. Los estudios han estado orientados a evaluar el éxito o fracaso de los proyectos de implementación tecnológica y han encontrado un alto índice de fallas como desproporciones en el presupuesto, retraso e incumplimiento de requisitos de acuerdo con lo solicitado al inicio. Estos aspectos se convierten en una amenaza para la continuidad de las empresas dedicadas a este sector (Serrador y Turner, 2015).

El McKinsey Global Institute (MGI) encontró en el año 2012 que, en promedio, los grandes proyectos de implementación tecnológica se ejecutaron en un $45 \%$ por encima del presupuesto con un $7 \%$ de tiempo adicional, y proporcionaron un $56 \%$ menos de ganancias. Por su parte, el Grupo Standish informó en 2014 que solo el 12 \% de los proyectos habían finalizado a tiempo y dentro del presupuesto (Bloch et ál., 2016).

Con base en estudios de la gestión de proyectos, metodologías como el Project Management Body of Knowledge (PMBOK) han sido de gran utilidad en la ejecución de proyectos para reducir tiempos de entrega. tal como lo ratifican estudios como el de Claro Perú (Moncada, 2018). En dicho documento, se determina la consecución de mejoras aplicando las técnicas desarrolladas por el Project Management Institute (PMI), organización dedicada a la administración y dirección de proyectos, donde los resultados han demostrado que estas herramientas y las buenas prácticas contenidas en el PMBOK proporcionan un valor en la gestión de proyectos, lo que ayuda a optimizar recursos.

A nivel mundial, se utilizan herramientas o metodologías aplicadas a la gerencia de proyectos que se ajustan a la naturaleza de cada tipo de proyecto. Actualmente, la administración de proyectos de telecomunicaciones muestra que las organizaciones son dinámicas por naturaleza, lo que hace inevitable que sean competentes y logren reestructurarse fácilmente según las circunstancias del mercado. Por tal 
razón, es necesario aplicar metodologías gerenciales en el sector de las telecomunicaciones (Cárdenas, 2007).

De la aplicación de metodologías gerenciales en el contexto nacional, se destaca el trabajo de grado sobre el diseño de una metodología para la gestión de proyectos de inversión en el Instituto Tecnológico Metropolitano (ITM). A pesar de que los proyectos en Colombia forman parte de una herramienta importante en la administración de organizaciones e instituciones públicas y privadas, no se tiene un registro de la gestión de proyectos y no hay un desarrollo en lo relacionado con la implementación de proyectos. Por tal motivo, la formulación y evaluación de estos es deficiente a nivel operativo y, "en consecuencia, es necesario que en Colombia se formulen acciones tendientes a actualizar las metodologías de gestión de proyectos" (Díaz y Carmona, 2011, p. 31). En esta misma línea, se plantea el desarrollo de las fases del proyecto aplicando la guía del PMBOK, lo que facilita el logro de los objetivos propuestos durante su ejecución (Jaimes, 2016).

En un estudio de Arévalo et ál. (2016), la empresa SKI partió de un requerimiento de su cliente Claro Soluciones Móviles para la implementación de 100 sitios indoor VIP en la ciudad de Bogotá en 6 meses. Para su ejecución, las áreas involucradas fueron integradas por medio de la guía del PMBOK en cuanto a la planeación y organización de las actividades solicitadas.

En otro contexto, la aplicabilidad de la guía PMBOK se valida con la práctica documentada por Vergara y Useche (2014), en la cual se implementó un sistema distribuido de antenas en el edificio de DirectTV, ubicado en la calle 103 de Bogotá, con el fin de mejorar la cobertura de telefonía móvil en las tecnologías GSM y UMTS. En este caso, se desplegaron todas las etapas requeridas por la guía PMBOK para este tipo de proyectos. 
Algunas organizaciones ni siquiera conocen la gestión de proyectos o no la reconocen como una estrategia funcional; por tal motivo, no están al tanto de los beneficios de la disciplina de la gestión de proyectos (SantiagoGuevara et ál., 2013).

Es importante mencionar que el sector de las telecomunicaciones está en una incesante evolución que ha transformado la economía de todo un país. En un comienzo, se podía considerar como un mercado estático, por cuanto la población demandaba servicios de telefonía fija, una tecnología con una infraestructura definida. A partir de la revolución de las telecomunicaciones móviles y la era de la información, las necesidades de la sociedad comenzaron a desarrollarse y a cambiar, por lo cual, para mantener su cuota de mercado, los operadores de servicios de telefonía móvil han generado nuevas propuestas a fin de dar soluciones a los usuarios de manera efectiva.

La gestión de proyectos no está por fuera de este proceso de cambios y se han generado procesos genéricos que permiten su aplicación en cualquier tipo de proyectos, aunque es complicado encontrar un plan, metodología o documento oficial que se ajuste a la naturaleza de cada proyecto.

Por tal razón, dentro del marco teórico se definen los conceptos fundamentales que hacen parte de la temática. En primer lugar, la tecnología $3 \mathrm{G}$ adoptada en el país corresponde a UMTS (universal mobile telecommunications system). Es una tecnología móvil que representa la evolución de GSM, GPRS y EDGE. Su interfaz aérea se beneficia de la eficiencia espectral de CDMA (code division multiple access), que para el caso de UMTS utiliza un ancho de banda de $5 \mathrm{MHz}$, por lo que también se conoce como WCDMA (Wideband CDMA). Esta tecnología soporta la integración de servicios de voz y datos, con tasas de transmisión en la interfaz de radio que van desde $384 \mathrm{kbps}$ en espacios abiertos y hasta 2 Mbps en áreas de baja movilidad en espacios interiores de edificios de 
acuerdo con el release 99 de la 3GPP (Torres, 2005). En segundo lugar, se tiene una red de nodos (antenas) separados espacialmente que permiten la ampliación de cobertura en áreas específicas, tales como un edificio, un centro comercial, un hospital, un estadio o un aeropuerto.

Cabe destacar que este tipo de sistemas puede operar en distintas bandas, estándares y operadores. Este es un tipo de solución dedicada que puede ser de gran utilidad para los operadores, ya que les permite brindar a sus clientes calidad del servicio y ampliar la cobertura de una determinada estación base (Durango y Gabriel, 2018).

Por último, de acuerdo con el PMI, una metodología de planeación de un proyecto es un proceso estructurado que se utiliza para guiar al equipo de administración del proyecto durante su ejecución. Esto incluye una variabilidad en la complejidad, por cuanto puede abarcar solo formatos o plantillas que pueden ser aplicados modelando situaciones y escenarios paso a paso con todas sus posibles interrelaciones (PMI, 2017). Profundizando en el contexto, "la literatura indica que una metodología tiene el fin de gestionar un proyecto de manera preventiva y no reactiva" (Sequeira, 2010, p. 27).

En esta investigación se estudiaron las causas del problema que enfrentaban las empresas de telecomunicaciones móviles, con un enfoque en la gestión de proyectos de implementación de sistemas distribuidos de antenas. Se partió de un diagnóstico inicial con expertos en el área con el fin de determinar el nivel de adopción de las metodologías gerenciales en los proyectos de implementación de sistemas distribuidos de antenas. Luego, se desarrolló una metodología apropiada para el control de la implementación de un sistema distribuido de antenas, identificando cada uno de los objetivos clave y considerando los factores de riesgo durante las fases de ejecución. Esto permitió monitorear el progreso en cada etapa para reducir las posibles desviaciones en el cumplimiento de los objetivos 
del plan, teniendo en cuenta las falencias identificadas en los resultados de las encuestas, los procesos adecuados para cada área y el manejo de las herramientas requeridas.

\section{Metodología}

A continuación, se describen los procedimientos utilizados para recolectar la información necesaria en el diseño de la metodología, y el análisis de las áreas del conocimiento en las diferentes técnicas, métodos, herramientas y guías de gerencia de proyectos, cuyas definiciones son primordiales para la buena administración de un proyecto.

Se determinó la presente investigación por modalidades de exploración, con diseño de investigación documental, basada en datos pertenecientes a la realidad. Esta modalidad de investigación permite realizar consultas en referencias bibliográficas, artículos, revistas, libros electrónicos, entre otros, mediante las que se identificaron técnicas que ayudaron a solucionar la metodología de gerencia de proyectos en sistemas distribuidos de antenas.

La recolección de datos se realizó mediante encuestas aplicadas a personal experto, ingenieros y técnicos, con conocimiento de los sucesos ocurridos de forma inesperada que afectan de manera significativa los cumplimientos. Teniendo en cuenta las fuentes consultadas, se seleccionaron variables como marco de referencia para elaborar una metodología de gestión de proyectos y se planteó el uso de técnicas cuantitativas que ayudaron a evaluar los procedimientos, las prácticas técnicas y la ejecución de proyectos de sistemas distribuidos de antenas. El muestreo para esta investigación fue no probabilístico con procedimiento por conveniencia para la recolección de información. Se realizó una tabulación de datos y luego un análisis minucioso de los 
resultados para definir las fases y los elementos fundamentales de la metodología gerencial aplicable a este tipo de proyectos de implementación de sistemas distribuidos de antenas. Al finalizar esta etapa, se estudiaron diferentes metodologías gerenciales y de gestión de proyectos aplicables en el desarrollo de actividades de sistemas tecnológicos que implican un cambio constante por la revolución tecnológica, la cual requiere estar actualizados en temas de infraestructura y evitar contratiempos que generen doble esfuerzo.

De una forma sencilla, se ejecutaron las actividades o procesos en un orden lógico y consecutivo para tener la organización de todo lo que implica terminar un producto o servicio, por lo cual se observaron las prácticas utilizadas en la finalización del proyecto y se obtuvieron buenos resultados para todos los involucrados. Se evaluó este planteamiento paso a paso y se identificaron los momentos en los que la dinámica de funcionamiento fallaba. De igual forma, se buscó mitigar el riesgo que involucraba la ejecución de este tipo de proyectos.

Con la información organizada, valorada y evaluada, se procedió a plantear la metodología gerencial que se ajusta a los sistemas distribuidos de antenas, la cual ayudó a reducir todos los inconvenientes que se presentaban a diario cuando se ejecutaba la implementación.

De esta manera, se ejecutó cada uno de los procedimientos. En primera instancia, se aplicaron encuestas a personas que trabajan en empresas de telecomunicaciones donde se ejecutan proyectos de sistemas distribuidos de antenas. En la aplicación del instrumento, se incluyeron seis personas del área técnica, diez personas del área de ingeniería y ocho expertos del equipo gerencial.

Se identificó la muestra con una población objetivo de hombres y mujeres técnicos, profesionales o gerentes de proyectos que trabajan en la 
implementación de sistemas distribuidos de antenas en empresas del sector de telecomunicaciones de Bogotá. Se eligió la técnica de muestreo no probabilístico por conveniencia. Las muestras de este tipo utilizan un método de selección orientada.

La ventaja de una muestra no probabilística desde el enfoque cuantitativo "es su utilidad para determinados diseños de estudio que requieren no tanto una 'representatividad' de elementos de una población, sino una cuidadosa y controlada elección de casos con ciertas características especificadas previamente en el planteamiento del problema" (Hernández et ál., 2013, p. 190).

\section{Diseño del instrumento}

El propósito del instrumento fue recolectar información para identificar los factores de alta criticidad en la implementación de sistemas distribuidos de antenas de tecnología de 3G. Se realizaron tres tipos de encuesta para tener una evaluación integral de la situación de este tipo de proyectos.

\section{Aplicación del instrumento}

Las encuestas se aplicaron al personal de ocho empresas del sector de telecomunicaciones ubicadas en Bogotá. Máximo tres empleados por organización podían responder el instrumento. A continuación, se detallan los resultados de la aplicación.

\section{Resultados y discusión}

En su totalidad, los encuestados están de acuerdo en que la implementación de sistemas distribuidos de antenas es necesaria para los usuarios. Con este resultado se confirma la necesidad de ejecutar estos proyectos, lo que implica la administración de proyectos (figura 1). 
Figura 1. Pregunta 1

1. ¿La implementación de sistemas distribuidos de antenas es necesaria para los us uarios?

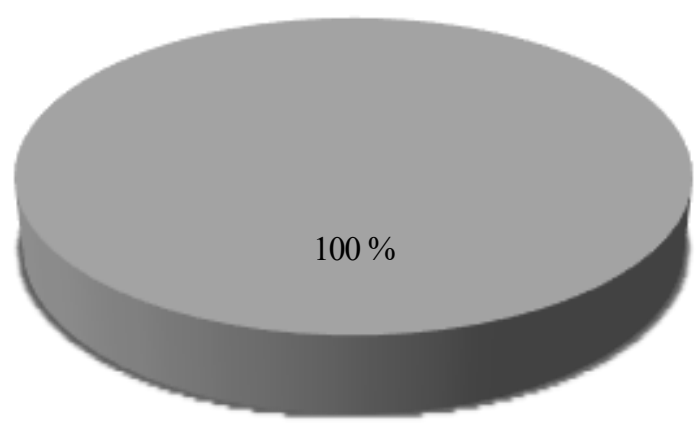

Sí $=$ No

Fuente: elaboración propia.

Por su parte, en la pregunta 2 (figura 2), el $100 \%$ de los encuestados indica que se pueden mejorar los tiempos de ejecución de los sistemas distribuidos de antenas. El personal técnico afirma que se pueden optimizar los tiempos, lo que señala una alta probabilidad de evaluar y aplicar procesos que permitan reducir los tiempos. 
2. ¿Cree usted que se puedan mejorar los tiempos de ejecución en este tipo de soluciones?

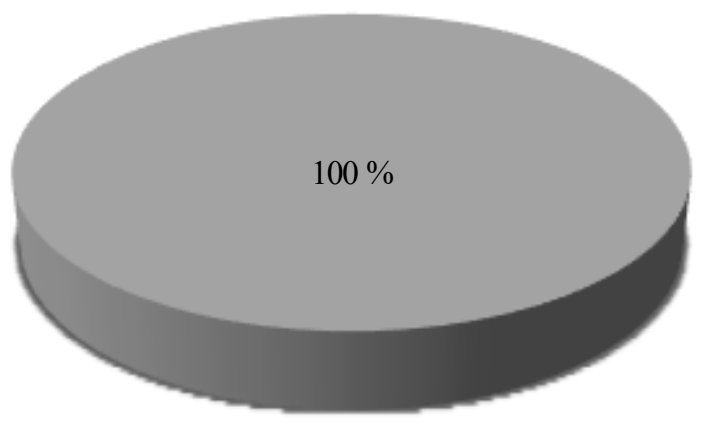

$\square$ Sí $\square$ No

Fuente: elaboración propia.

Para complementar esta pregunta, se solicitó que, en caso de que la respuesta fuera afirmativa, el encuestado indicara cuáles alternativas consideraría apropiadas para la optimización de estos tiempos, como se muestra en la figura 3. De acuerdo con las respuestas a esta pregunta, se obtuvieron las siguientes alternativas:

- Una buena coordinación (permisos, ingresos, conocimiento del trabajo por parte del personal, material completo para realizar el trabajo, coherencia con los tiempos establecidos para la ejecución de los trabajos) y conocimiento del trabajo por parte de todo el personal (coordinadores, técnicos, supervisores, etc.).

- Mejorar la comunicación entre las partes que intervienen en el proceso y mayor socialización para los encargados de las estructuras o edificaciones, según sea el caso.

- Tener muy claro lo requerido para la implementación y que todo se pueda hacer en conjunto.

- Coordinación y cumplimiento. 
- Coordinar con el contratista y mantener informado al cliente de lo que se va a hacer.

Figura 3. Pregunta 2

En caso de que su respuesta sea afirmativa, ¿qué alternativas consideraría apropiadas para la optimización de es tos tiempos?

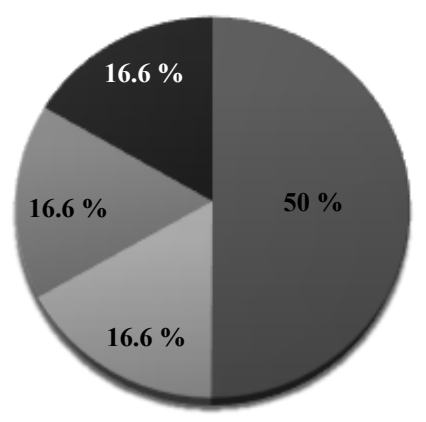

Una buena coordinación (permisos, ingresos, conocimiento del trabajo por parte del personal, material completo para realizar el trabajo, coherencia con los tiempos establecidos para la ejecución de los trabajos), conocimiento del trabajo por parte de todo.

$\square$ Mejorar en la comunicación entre las partes que intervienen en el proceso y mayor socializacion de cara a los encargados de las estructuras o edificaciones según sea el caso.

$\square$ Tener muy claro lo requerido para la implementacion y que todo se pueda hacer en conjunto.

a No responde.

Fuente: elaboración propia.

El $50 \%$ de los encuestados está de acuerdo en que una de las alternativas para optimizar los tiempos es tener una buena coordinación de todas las actividades requeridas para la ejecución. El 33 \% indica que se pueden mejorar los tiempos de ejecución de los sistemas distribuidos de antenas al tener un proceso adecuado de comunicación y conocer los requerimientos, mientras que el $17 \%$ no responde a la pregunta.

La gran mayoría de los encuestados coinciden en que se deben mejorar los procesos y las funciones correspondientes a la coordinación de las actividades, y se deben organizar de acuerdo con la secuencia de estas durante la implementación.

La figura 4 muestra los hallazgos de la pregunta 3 . El $60 \%$ de los encuestados indica que el cumplimiento de los objetivos depende 
principalmente de la aplicación de una metodología y el conocimiento de los objetivos propuestos, mientras el $40 \%$ indica que el cumplimiento de los objetivos obedece al compromiso del equipo de trabajo.

Figura 4. Pregunta 3

3. Estima usted que el cumplimiento de los objetivos depende principalmente de:

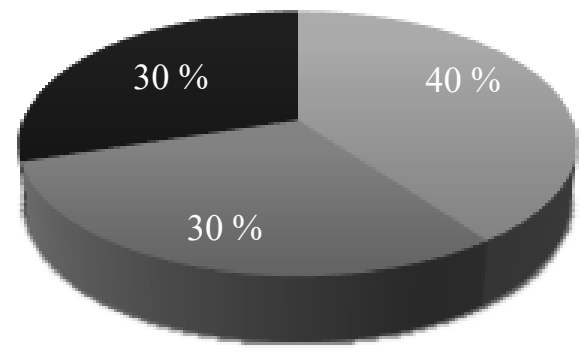

Gerente del proyecto

Compromiso del equipo de trabajo

Aplicación de una metodología

- Conocimiento y claridad de los objetivos propuestos

Otro

Fuente: elaboración propia.

Se puede afirmar que para lograr los objetivos es necesario aplicar una metodología que permita divulgar los objetivos del proyecto y mejorar la comunicación de todos los miembros del área de ingeniería, con el propósito de que todos conozcan sus responsabilidades y adquieran un compromiso con el cumplimiento de los objetivos.

La siguiente pregunta se detalla en la figura 5 . El $32 \%$ de los encuestados indica que el mayor inconveniente en la ejecución de los proyectos es cumplir con el tiempo asignado, para el $26 \%$ consiste en no tener claro el alcance, para el $16 \%$ el proceso de comunicación es inadecuado, para un 
$11 \%$ el problema es el costo del proyecto, que termina siendo superior al presupuestado, y para otro $11 \%$ es la carencia de un adecuado seguimiento y control. El $5 \%$ restante dice que no se tienen en cuenta todos los interesados del proyecto.

Figura 5. Pregunta 4

4. ¿Cuál considera que es el mayor inconveniente en la ejecución o gerencia de los proyectos que ha manejado?

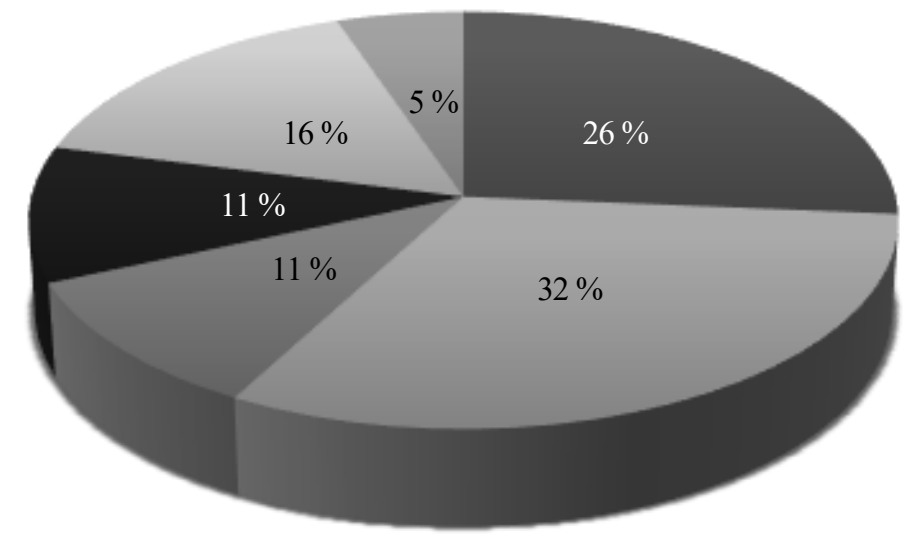

El alcance no es claro

- Tiempos que no se pueden cumplir

Costo superior al presupuestado

- No se tienen seguimiento ni control

$\square$ Comunicación inadecuada

Otra (no se tienen en cuenta todos los interesados en el proyecto)

Fuente: elaboración propia.

A partir de estas respuestas, se puede afirmar que los elementos críticos a la hora de ejecutar un proyecto de sistemas distribuidos de antenas son el tiempo, el alcance y la comunicación. Es pertinente tener en cuenta estos aspectos a la hora de establecer una metodología gerencial aplicable a esta área. Intervenir estos procesos es fundamental para mejorar los factores críticos en este tipo de soluciones, por lo cual se debe determinar el proceso adecuado con su respectiva herramienta que permita hacer seguimiento. 
La figura 6 muestra los resultados de la pregunta 5. El 62.5\% de los encuestados señala que se ha realizado una acción correctiva para los imprevistos que se presentan en el desarrollo de los proyectos; el $37.5 \%$ restante dice que no ha realizado ninguna acción correctiva. Para las respuestas afirmativas, se consideraron las siguientes alternativas: crear listas de chequeo para control documental y de seguimiento; validar actividades finalizadas para incluir más personal en lo que resta del proyecto; realizar modificaciones en el producto para cumplir con tiempos y costos; tener más control sobre los colaboradores o aliados que ejecutan los proyectos; buscar una nueva solución para cumplir con el proyecto; dejar plasmadas en el cronograma todas las actividades relacionadas con la comunicación y el buen funcionamiento cuando el sistema debe comunicarse con un aplicativo externo.

Figura 6. Pregunta 5 5. ¿Se ha realizado alguna acción correctiva para los imprevistos presentados
en el desarrollo de los proyectos?

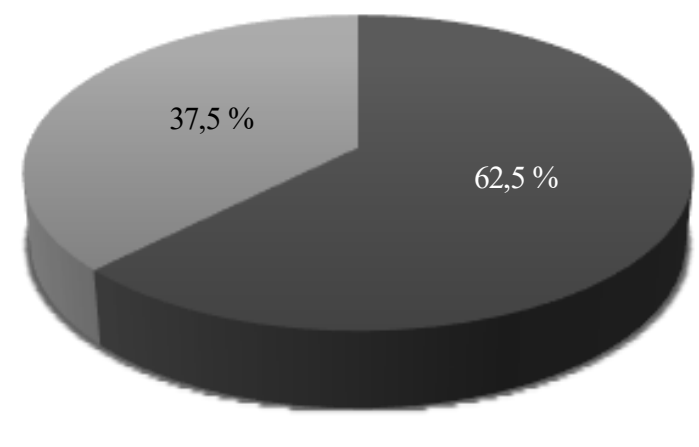

घ Sí $\square$ No

Fuente: elaboración propia.

En conexión con el interrogante anterior, la figura 7 presenta los resultadoas de la pregunta 6 . 
Figura 7. Pregunta 6

6. ¿Cree que es suficiente esta acción correctiva para obtener los resultados esperados?

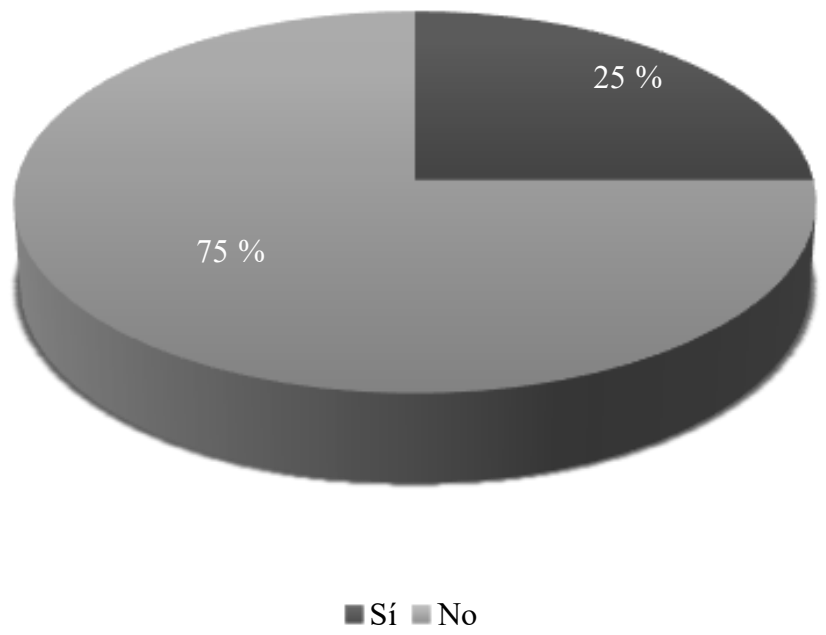

Fuente: elaboración propia.

El $75 \%$ de los encuestados indica que no es suficiente la acción correctiva para obtener los resultados esperados y el $25 \%$ restante dice que sí es suficiente. En el caso de las respuestas afirmativas, se planteó evaluar el cumplimiento del proyecto en los siguientes aspectos:

- $\quad$ Objetivos estratégicos

- $\quad$ Alcance del proyecto

- Tiempo

- $\quad$ Calidad

- $\quad$ Costos

Finalmente, la figura 8 muestra información adicional de la pregunta 6, en caso de que la respuesta anterior fuera afirmativa: 
Figura 8. Pregunta 6



Fuente: elaboración propia.

De acuerdo con las respuestas obtenidas, se puede afirmar que las acciones correctivas ejecutadas no son efectivas en su gran mayoría y solo en pocos casos son una solución para cumplir con los objetivos estratégicos, el alcance, la calidad, los costos y el tiempo del proyecto. En este sentido, las acciones correctivas no garantizan los resultados y pueden variar. Por tal motivo, se requiere aplicar una metodología que ayude a prever, mitigar y corregir de manera correcta las eventualidades que pueden generar impactos negativos en la ejecución de los proyectos.

Después de analizar los resultados de la aplicación de las encuestas, se concluye que es necesario implementar sistemas distribuidos de antenas. Sin embargo, existen dificultades en el proceso de ejecución en lo que respecta a los tiempos, donde los encuestados manifiestan alternativas para tratar las áreas que presentan mayores dificultades, las cuales no han sido solucionadas con las acciones correctivas desarrolladas. Se detallan aspectos en los que se presentan mayores incidencias, es decir, los 
elementos o factores críticos que deben considerarse en el diseño de la metodología. En la tabla 1 se relacionan los puntos a intervenir.

Tabla 1. Identificación de elementos críticos en proyectos de implementación de sistemas distribuidos de antenas

\begin{tabular}{|l|l|l|}
\hline \multicolumn{1}{c}{$\begin{array}{c}\text { Elementos } \\
\text { críticos }\end{array}$} & \multicolumn{1}{|c}{ Descripción } & \multicolumn{1}{c|}{ Causas } \\
\hline Coordinación & $\begin{array}{l}\text { En la ejecución se presentan muchos } \\
\text { inconvenientes en la coordinación (permisos, } \\
\text { ingresos, entrega de materiales). }\end{array}$ & $\begin{array}{l}\text { Planificación del proyecto } \\
\text { (no se lleva un registro } \\
\text { del trabajo y las } \\
\text { actividades requeridas) }\end{array}$ \\
\hline Tiempo & $\begin{array}{l}\text { No hay coherencia en los tiempos establecidos } \\
\text { para la ejecución del proyecto. } \\
\text { Desconocimiento por parte del personal técnico } \\
\text { del cronograma. }\end{array}$ & $\begin{array}{l}\text { Cronograma (no tiene el } \\
\text { detalle de actividades.) }\end{array}$ \\
\hline Calidad & $\begin{array}{l}\text { El producto entregado no es el esperado por } \\
\text { cliente. }\end{array}$ & $\begin{array}{l}\text { Desconocimiento de los } \\
\text { requisitos y } \\
\text { requerimientos del } \\
\text { cliente }\end{array}$ \\
\hline Comunicación & $\begin{array}{l}\text { Comunicación inadecuada entre las partes que } \\
\text { intervienen en el proceso, de manera que se } \\
\text { presentan novedades en el desarrollo de las } \\
\text { actividades por falta de información y } \\
\text { socialización. }\end{array}$ & $\begin{array}{l}\text { Gestión de las } \\
\text { comunicaciones (no está } \\
\text { definido el proceso de } \\
\text { comunicación.) }\end{array}$ \\
\hline
\end{tabular}

Fuente: elaboración propia.

La metodología propuesta cuenta con cinco fases (figura 9) que intervienen en cuatro áreas fundamentales donde se identificaron los factores críticos (coordinación, tiempo, calidad y comunicación). Se manejan los procesos de la gestión gerencial en la implementación de sistemas distribuidos de antenas cuyo objetivo es mejorar los métodos para la satisfacción de los clientes.

De acuerdo con este modelo, se intervienen cuatro áreas fundamentales (gestión de la integración, gestión del tiempo, gestión de la calidad y gestión de las comunicaciones e información). Con la primera se integran los cinco procesos (inicio, planificación, implementación, seguimiento y control, cierre) durante el desarrollo de la implementación. Aunque con una sola guía como el PMBOK se lograba abordar todas las áreas, se 
eligieron cada una de las herramientas por sus criterios de autenticidad y sencillez para aplicar en los procesos a intervenir. Se incorporaron los aportes valiosos de PMBOK (Project Management Institute, 2017), International Project Management Association (2015), ISO 21500 y P2M.

Figura 9. Estructura de la metodología propuesta

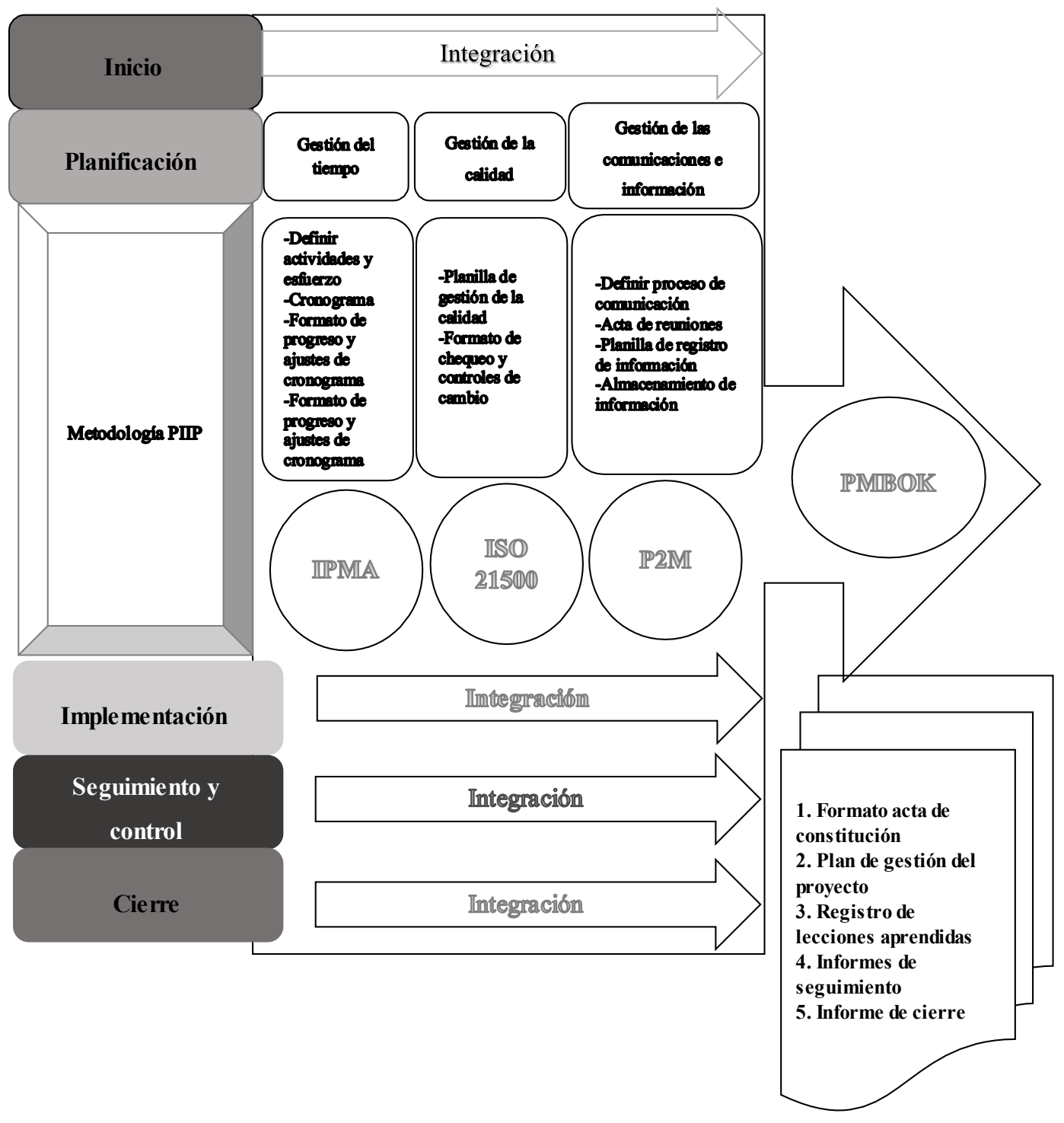

Fuente: elaboración propia. 


\section{Gestión de la integración}

Inicio

Formato acta de constitución. Visita de preinstalación al sitio, reunión de socialización de los resultados de la visita de preinstalación (clienteempresa instaladora), socialización del proyecto con el personal técnico, validación de la visita de preinstalación, proceso para los trámites de ingreso y horarios para ejecutar las actividades y verificación de materiales disponibles para dar inicio a la implementación. Se concreta con la administración del centro comercial la asignación de bodega en sótano para el almacenamiento de materiales y herramientas.

\section{Planificación}

Se definen y secuencian las actividades, se realiza cronograma y se formaliza el seguimiento y control del cronograma durante la implementación.

Implementación

Registro de lecciones aprendidas (formato de lecciones aprendidas).

\section{Seguimiento y control}

Informes de desempeño (plantilla de seguimiento).

\section{Cierre}

Informe de cierre (formato de cierre).

Gestión del tiempo

Se definen las actividades, el cronograma, el formato de progreso y los ajustes (formatos). 


\section{Gestión de la calidad}

Plantilla de gestión de calidad, formato de chequeo y controles de cambios (formatos). Al tener definido el plan para la gestión de calidad, se determina un indicador de calidad del proceso o actividad en la fase de implementación para tener el nivel de cumplimiento en la realización de las actividades y poder plasmar en una matriz de política de calidad.

\section{Gestión de comunicaciones e información}

Se define el proceso de comunicación, el acta de reuniones, la plantilla de registro de información y el almacenamiento de la información (formatos). Se determina el proceso de comunicación, se informa a los miembros del equipo el proceso de comunicación, se establece una unidad para almacenar la información (actas, contratos, planos, pruebas, registro fotográfico, entre otros). Cabe mencionar que cada empresa debe definir dónde será la recopilación.

Después de la aplicación de la metodología propuesta, en cada una de las fases de la ejecución de un sistema distribuido de antenas, el gerente del proyecto manifiesta que la organización de las fases del proyecto permite controlar el desarrollo de cada una de las actividades, pues cuando no se aplicaba una metodología, era difícil conocer el estado real de los avances del proyecto. Se cumplió con los tiempos estipulados para la implementación en proyectos anteriores y se evitó así pagar multas por el incumplimiento con las fechas de finalización y entrega.

Por otra parte, se desarrolló un proceso de comunicación e información de manera sencilla, clara y efectiva, de tal manera que al cliente le fueron entregados de forma oportuna los informes de avances y reportes de novedades. Cuando no se utilizaba la metodología, algunas cosas se 
comunicaban y otras no; en ocasiones no se hacía de inmediato, lo que causaba molestias al cliente.

Por último, se obtuvo un producto de calidad que cumple con los requerimientos iniciales, teniendo en cuenta que en otras circunstancias se presentaban novedades en la entrega parcial, por lo que el cliente quedaba inconforme por detalles finales de la instalación. Esto implicaba hacer un nuevo envío para hacer las mejoras y correcciones pertinentes. Sin embargo, la aplicación de la metodología aportó mejoras significativas en los procesos intervenidos.

\section{Conclusiones}

Se desarrolló una metodología con lineamientos para la implementación de sistemas distribuidos de antenas, que fue aplicada en la ejecución de un proyecto para intervenir las áreas de coordinación, tiempo, calidad y comunicación. Asimismo, se validó la funcionalidad de cada uno de los procesos planteados y se observaron mejoras en todas las fases desde el inicio hasta el final.

Todo esto fue posible al identificar las variables que inciden en la implementación de este tipo de soluciones y la aplicabilidad de las diferentes metodologías gerenciales. Con esta información, se propuso una metodología gerencial práctica y ajustada a la naturaleza de estos sistemas. Esta herramienta facilita el seguimiento y el control de las actividades requeridas durante el desarrollo de las diferentes etapas, lo que proporciona al gerente del proyecto una guía que garantiza el cumplimiento de los objetivos y la satisfacción del cliente. Este estudio es una base para futuros trabajos de metodologías gerenciales y para la implementación de sistemas distribuidos de antenas en la innovación de nuevas tecnologías. 


\section{Referencias}

Arévalo, D., Bernate, N. y Pardo, F. (2016). Implementación 100 sitios indoor de comunicación $3 G$ y $4 G$ [Tesis de posgrado]. Universidad Santo Tomás de Aquino.

Bloch, M., Blumberg, S. y Laartz, J. (2012). Delivering large-scale IT projects on time, on budget, and on value. McKinsey on Business Technology, 27, 2-8. https://mck.co/2KGujg1

Cárdenas, M. (2007). Metodología de gerencia por procesos, para proyectos de implementación de equipos en el área de telecomunicaciones [Tesis de maestría]. Escuela Politécnica Nacional.

Díaz, C. y Carmona, P. (2011). Diseño de una metodología para la gestión de proyectos de inversión en el ITM, basada en el Project Management Institute-PMI [Tesis de maestría]. Universidad de Medellín.

Durango, G. (2018). Diseño de un sistema distribuido de antenas celulares para el túnel del Cerro El Carmen de Guayaquil utilizando el cable SCTP CAT 5/6 para la banda GSM $850 \mathrm{MHz}$ [Tesis de pregrado]. Escuela Superior Politécnica del Litoral.

Garzón, H. (2013). Guía de gerencia de proyectos. XVII Foro Mundial de la Calidad y de la Gestión para la Mejora INLAC 2013, Veracruz, México.

Hernández, R., Fernández, C. y Baptista, L. (2013). Metodología de la investigación (6. a ed.). McGraw Hill.

International Project Management Association. (2015). Individual competence baseline for project, programme \& portfolio management. Version 4.0. https://www.pma.at/files/downloads/440/ipmaicb4.pdf

Jaimes, F. (2016). Propuesta de diseño e implementación de un sistema distribuido de antenas (DAS) para mejorar la red móvil del operador claro en el centro comercial titán plaza [Tesis de Pregrado]. Universidad Distrital Francisco José de Caldas.

Joslin, R. y Müller, R. (2015). Relationships between a project management methodology and project success in different project governance contexts. International Journal of Project Management, 33, 1377-1392.

https://doi.org/10.1016/j.ijproman.2015.03.005

SIGNOS, Investigación en Sistemas de Gestión

ISSN: 2145-1389 | e-ISSN: 2463-1140 | DOI: https://doi.org/10.15332/24631140

Vol. 13 N.o 1 | enero-junio de 2021 
Moncada, I. (2018). Gestión de proyectos para mejorar el plazo de entrega de proyectos en la empresa de telecomunicaciones "claro" [Tesis de pregrado]. Universidad Nacional de Trujillo.

Neira, L. (2019, 12 de junio). La 'papa caliente' de la ministra de las TIC, Silvia Constaín, con la dominancia. La República. https://www.larepublica.co/empresas/la-papacaliente-de-la-ministra-de-las-tic-silvia-constain-con-la-dominancia-2872754/

Project Management Institute. (2017). La guía de los fundamentos para la dirección de proyectos (Guia del $P M B O K 囚)$ (6. ${ }^{\mathrm{a}}$ ed.).

Santamaría, J., y Jiménez, M. (2013). Propuesta de metodología para la gestión de proyectos de alianzas estratégicas en la división banda ancha del sector telecomunicaciones del ICE a fin de brindar soluciones de tercerización a clientes empresariales [Tesis de maestría]. Instituto Tecnológico de Costa Rica.

Santiago-Guevara, J. C., Rojas-Contreras, W. M. y Esteban-Villamizar, L. A. (2013). Gestión de comunicaciones en los proyectos. Tecno Lógicas, Edición Especial, 465479. https://doi.org/10.22430/22565337.347

Sequeira, W. (2010). Metodología para administración de proyectos del departamento de desarrollo de la compañía automatización avanzada [Tesis de Maestría]. Universidad para la Cooperación Internacional.

Serrador, P. y Turner, J. R. (2015). The relationship between project success and project efficiency. Procedia-Social and Behavioral Sciences, 46(1), 30-39.

https://doi.org/10.1002/pmj.21468

Torres, J. (2005). Diseño de una celda celular UMTS para la Escuela Politécnica Nacional [Tesis de pregrado]. Escuela Politécnica Nacional.

Useche, G. y Vergara, Y. (2014). Ampliación de cobertura telefonía móvil, tecnología UMTS y GSM con diseño inbuilding en oficinas principales edificio DirecTV Bogotá calle 103 plaza [Tesis de posgrado]. Universidad Santo Tomás. 